\title{
BMJ Open Opioid use and harms associated with a sustained-release tapentadol formulation: a postmarketing study protocol
}

\author{
Amy Peacock, ${ }^{1,2}$ Briony Larance, ${ }^{1}$ Michael Farrell, ${ }^{1}$ Rose Cairns, ${ }^{3,4}$ \\ Nicholas Buckley, ${ }^{3,4}$ Louisa Degenhardt ${ }^{1,5,6,7}$
}

To cite: Peacock A, Larance B, Farrell $\mathrm{M}$, et al. Opioid use and harms associated with a sustained-release tapentadol formulation: a postmarketing study protocol. BMJ Open 2018;8:e020006. doi:10.1136/ bmjopen-2017-020006

- Prepublication history and additional material for this paper are available online. To view these files, please visit the journal online (http://dx.doi. org/10.1136/bmjopen-2017020006).

Received 9 0ctober 2017 Revised 12 February 2018 Accepted 15 February 2018

Check for updates

${ }^{1}$ National Drug and Alcohol Research Centre, University of New South Wales, Sydney, New South Wales, Australia

${ }^{2} S$ chool of Medicine, University of Tasmania, Hobart, Tasmania, Australia

${ }^{3}$ NSW Poisons Information Centre, The Children's Hospital at Westmead, Sydney, New South Wales, Australia

${ }^{4}$ School of Medical Sciences, University of Sydney, Sydney, New South Wales, Australia ${ }^{5}$ School of Population and Global Health, University of Melbourne, Melbourne, Victoria, Australia ${ }^{6}$ Murdoch Children's Research Institute, Parkville, Victoria,

Australia

${ }^{7}$ Department of Global Health, School of Public Health, University of Washington, Seattle, Washington, USA

Correspondence to

Dr Amy Peacock;

Amy.Peacock@unsw.edu.au

\section{ABSTRACT}

Introduction It has been argued that tapentadol may pharmacologically have lower abuse potential than other pharmaceutical opioids currently available. However, there has been no comprehensive triangulation of data regarding use and harms associated with this formulation. $A$ sustained-release formulation (SRF) of tapentadol (Palexia) was released in Australia in 2011 and listed for public subsidy in 2013. We summarise here the methods of a postmarketing study which will measure postintroduction: (1) population level availability, (2) extramedical use and diversion, (3) attractiveness for extramedical use and (4) associated harms, of tapentadol compared against other pharmaceutical opioids.

Methods and analysis We evaluated key sources on pharmaceutical use and harms in Australia. This review indicateddata from four sources that disaggregate pharmaceutical opioid formulations and capture tapentado SRF could be triangulated. These data sources comprised: (1) national pharmaceutical opioid community sales data from 2011 to 2017, (2) national pharmaceutical opioid poisonings reported to Poison Information Centres (PICs) from 2011 to 2017, (3) number of vendors on online marketplaces listing pharmaceutical opioids for sale and (4) data on pharmaceutical opioid extramedical use, attractiveness and harms from interviews with people who regularly inject drugs in Australia.

Ethics and dissemination Ethics approval is not required for use of pharmaceutical sales data. Ethics approval has been obtained for use of national pharmaceutical opioid poisonings reported to PICs (LNR/16/SCHN/44) and for use of online marketplace data and interview data from people who inject drugs (HC12086). Key findings will be published mid-2018 in a peer-reviewed academic journal, and presented at various conferences and professional meetings.

\section{INTRODUCTION}

In the past two decades, there has been an increase in the number of pharmaceutical opioids available, and in the prescribing of these drugs, in several high-income countries, including North America and Australia. Indeed, between 1992 and 2012, the number of pharmaceutical opioid dispensing episodes increased 15-fold in Australia. ${ }^{2}$ This

\section{Strengths and limitations of this study}

This study will comprise the first published comprehensive assessment of tapentadol sustained-release formulation use, extramedical use, attractiveness for extramedical use and associated harms.

- The number of data sources is limited relative to other postmarketing studies of pharmaceutical opioids as tapentadol is rarely coded separate to other pharmaceutical opioids in administrative data.

- However, this study will overview a wealth of data from the general population and a group at high risk for extramedical use, both of which are key to examine when quantifying various impacts of pharmaceutical opioids.

- These findings will have relevance to other countries where tapentadol has recently been introduced or where it may be introduced in the future.

increase in availability in Australia has been accompanied by greater rates of extramedical use, defined as use outside the bounds of a doctor's prescription. ${ }^{3}$ The risk of opioid dependence and serious adverse events (eg, overdose) associated with extramedical use has made addressing this problem a public health imperative. ${ }^{45}$

Opioids differ in the extent to which they are likely to be associated with hazardous patterns of use due to different potencies (ie, weak to strong opioids), pharmacokinetic characteristics (eg, rate of metabolism) and propensities for dependence. ${ }^{6}$ The need to reduce extramedical use must also be balanced against the need for access to opioid therapy for pain. Thus, current public health agency strategies to reduce extramedical pharmaceutical opioid use and chronic pain prescribing guidelines focus on products which have limited or no abuse potential. ${ }^{78}$

Tapentadol is a centrally acting opioid analgesic with dual mechanisms of action, specifically $\mu$-opioid receptor agonist and norepinephrine reuptake inhibition. This dual action is thought to result in a lower 
dose required to produce a given level of analgesia. ${ }^{9}$ Clinical trials suggest that tapentadol provides equivalent or superior levels of pain relief for acute and chronic pain similar to oxycodone and morphine, with greater gastrointestinal tolerability in terms of reduced rates of nausea, vomiting and constipation. ${ }^{10-13}$

A recent review by the World Health Organization's Expert Committee on Drug Dependence ${ }^{11}$ concluded that potential for abuse for tapentadol may be similar to or slightly lower than other opioids such as hydromorphone, oxycodone, morphine and tramadol. However, they note that these conclusions are tentative given a lack of data regarding tapentadol 'abuse, dependence, diversion, recreational use or poison control', noting that tapentadol generally does not feature in drug use surveys or surveillance reports. The few studies which have been conducted are based on US samples, typically evaluating outcomes in using treatment-seeking populations ${ }^{14}$ or assessing internet discussions ${ }^{1516}$ and prescribing data ${ }^{17}$ todetermine extramedical use. Early indications of rates of abuse and diversion from these studies show equivalence to hydromorphone and lower than oxycodone and most other strong opioids. ${ }^{14} 18$

The immediate release formulation of tapentadol is registered in Australia for moderate to severe pain, while the sustained release formulation is registered for severe pain which requires constant opioid treatment and for which no other opioid other treatments are adequate. ${ }^{19}$ Although a sustained-release formulation of tapentadol (SRF; Palexia SR) was released in Australia in 2011, and listed for subsidy on the Pharmaceutical Benefits Scheme in November $2013,{ }^{20}$ no research into rates into dispensing, extramedical use and associated harms of the tapentadol SRF has been undertaken in Australia. Given the broader context of rising rates of pharmaceutical opioid use and harms (including overdose), monitoring new formulations in terms of population level pharmaceutical opioid use, and extramedical use among highrisk populations (eg, those reporting extramedical use of other pharmaceutical use), is critical.

This paper outlines the design for a national postmarketing study of use, extramedical use and harms associated with introduction of the tapentadol SRF in Australia. This study will integrate population and sentinel high-risk population data, including national pharmaceutical opioid sales data, interviews with people who inject drugs, and national opioid-related poisoning event data.

\section{METHODS AND ANALYSIS \\ Study aims}

The overarching aim of this study is to identify extramedical use and diversion of tapentadol SRF, attractiveness for extramedical use and associated levels of harms compared with these indicators for other pharmaceutical opioid use. The specific questions we aim to answer are as follows:
1. What is the population level availability of the tapentadol SRF in Australia relative to other pharmaceutical opioids?

2. Are there indications of extramedical use of the tapentadol SRF (specifically, non-prescribed use, use via routes that require tampering), and how does this compare to other pharmaceutical opioids?

3. What is the relative attractiveness for extramedical use (eg, street price) of the tapentadol SRF, and how does this compare to other pharmaceutical opioids?

4. Are there indications of associated harm with the tapentadol SRF (specifically, self-reported non-fatal overdose, as well as intentional poisoning, extramedical use and abuse as identified through Poison Information Centre (PIC) data), and how does this compare to other pharmaceutical opioids?

\section{Study design and setting}

This study is being conducted in Australia. There are three main components of this study: analyses of existing routine data sources (2011-2017); analyses of data from monitoring online drug marketplaces (2013-2017) and analyses of cross-sectional data collected from a highrisk sentinel group (people who inject drugs) participating in the Illicit Drug Reporting System (IDRS) in 2016.

Most indicator data sets in Australia do not routinely separate tapentadol from other pharmaceutical opioids, precluding any comparison of population-level availability (see online supplementary appendix 1 ). Five data sources were identified which could provide data specific to pharmaceutical opioid brand, thus allowing analysis of use and harms related to tapentadol SRF. Prescription monitoring programme data, although theoretically useful, only exist in one jurisdiction, where registration is voluntary and implementation is not complete or consistent, and utilisation of this programme irregular. Consequently, four data sources were included in this study: pharmaceutical opioid sales data; PIC call data; online drug marketplace data and interviews with people who inject drugs. Table 1 outlines the relevant outcome for each data source for each research question.

\section{Pharmaceutical opioid sales data}

A third-party access request to obtain pharmaceutical sales data in Australia has been approved by IMS Health. Requested data include all pharmaceutical opioid purchases through pharmaceutical wholesalers and manufacturers who sold direct to pharmacies between 2011 and 2017. Data will reflects community sales only, excluding sales to hospitals. However, due to the legal requirements for secure storage of pharmaceuticals in pharmacies, and monitoring and recording of opioids depending on their schedule listing, the number of packs sold over a 12-month period closely approximates the number used.

All opioids available in Australia that are indicated of pain will be included and coded according to the WHO's 
Table 1 Summary of the primary outcomes from each data source

\begin{tabular}{|c|c|c|c|c|}
\hline Research outcome & $\begin{array}{l}\text { Pharmaceutical opioid } \\
\text { sales data }\end{array}$ & $\begin{array}{l}\text { Interviews with people } \\
\text { who inject drugs }\end{array}$ & $\begin{array}{l}\text { Online drug } \\
\text { marketplace data }\end{array}$ & $\begin{array}{l}\text { Poisons Information } \\
\text { Centre calls }\end{array}$ \\
\hline \multicolumn{5}{|c|}{ Research question 1: exposure } \\
\hline \multicolumn{5}{|c|}{ Research question 2: extramedical use } \\
\hline \multicolumn{5}{|c|}{$\begin{array}{l}\text { Levels of extramedical } \\
\text { use (diverted) }\end{array}$} \\
\hline \multicolumn{5}{|l|}{ Injection } \\
\hline \multicolumn{5}{|l|}{ Snorting } \\
\hline \multicolumn{5}{|l|}{ Chewing } \\
\hline \multicolumn{5}{|c|}{$\begin{array}{l}\text { Perceived availability } \\
\text { (diverted) }\end{array}$} \\
\hline \multicolumn{5}{|c|}{ Research question 4: harms } \\
\hline \multicolumn{5}{|c|}{ Overdose-non-fatal } \\
\hline \multicolumn{5}{|l|}{$\begin{array}{l}\text { Intentional exposure } \\
\text { (intentional self- } \\
\text { poisoning, misuse } \\
\text { and abuse) }\end{array}$} \\
\hline
\end{tabular}

Fields filled with black indicate outcomes which can be measured using the given data source.

Anatomical Therapeutic Classification system (http:// www.whocc.no/atc_ddd_index/)

Code of A02A 'opioids' and A02B 'other analgesics and antipyretics'. This includes, but is not limited to, tapentadol (N02AX06), buprenorphine (N02AE01), prescription and over-the-counter codeine (N02BA51, N02BE51, N02AA59, R05DA04), dextropropoxyphene (N02AC04, N02AC54), fentanyl (N02AB03), hydromorphone (N02AA03), methadone (N02AC52), morphine (N02AA01) and oxycodone (N02AA05, N02AA55). Injectable formulations will be excluded, being mainly prescribed in hospitals, and formulations of methadone and buprenorphine used solely for the treatment of opioid dependence are excluded. However, overthe-counter codeine is captured in this data source. IMS Health data will be provided from January 2011 to December 2017 aggregated monthly by opioid formulation, brand, strength and geographic unit.

\section{PIC call data}

Four PICs in Australia, based in New South Wales, Queensland, Victoria and Western Australia, together provide nationwide, round-the-clock poisoning advice to healthcare professionals and members of the public. National data will be extracted regarding number of cases of pharmaceutical opioid exposure poisonings (including over-the-counter codeine) reported to PICs. Specifically, opioid-related calls will only be extracted where the exposure type/intent is coded as 'recreational', so as to identify exposures where pharmaceutical opioids were taken for its intoxicating effects. ${ }^{21}$ Where the intentional exposure subtype is not available or unclear, calls regarding opioids will undergo a free-text search for markers of extramedical use and abuse (defined here as use of a drug in a way or for a purpose outside intended medical use, eg, excess quantity, recreational use, use for non-approved purposes), then manually reviewed by PIC staff and recoded.

Unit-level data collected between January 2011 and December 2017 will be extracted. Data fields to be extracted include call date, age group, gender, state/ territory, dose, brand, active ingredients and route of exposure.

\section{Online drug marketplace data}

An existing surveillance system in Australia monitors availability and types of substances sold online via the darknet. ${ }^{22}{ }^{23}$ The darknet is accessible only via The Onion Router, software that enables anonymous communication. Cryptomarkets (markets operating on the darknet) have been accessed weekly since 2013 using a dedicated user account. Exhaustive snapshots of each accessible marketplace are taken, including details of vendor name, listing description and, where possible, country of origin. For this study, the number of vendors listing illicit pharmaceutical opioids for sale on cryptomarkets between January 2013 and December 2017 will be extracted by brand and by month. This data cannot provide any 
information about consumers who are buying drugs on the cryptomarkets, or any data on the total number of sales via these sites. However, it does yield timely and sensitive information regarding drug availability online.

\section{IDRS data}

The IDRS is a national illicit drug monitoring system, one part of which comprises annual interviews with approximately 900 people who inject drugs interviewed in each capital city in Australia. Participants are recruited using multiple methods, including advertisements in street press, newspapers, treatment agencies, needle and syringe programmes and peer referral. To be eligible to participate, participants need to be at least 17 years of age; have injected at least monthly during the 6 months preceding interview; and have been a resident for at least 12 months in the capital city in which they are interviewed. The interview is administered by trained research staff faceto-face at a time and location convenient to them, and participants receive \$AUD40 reimbursement.

The core quantitative interview monitors patterns of drug use and includes questions on price, purity and availability of the main drug types, including pharmaceutical opioids. ${ }^{24}$ Data will be used from the 2017 interview (conducted May-June 2017), which included additional items around tapentadol SRF use. These items pertained to:

- Levels of tapentadol extramedical use: diverted Palexia SR, swallowed, injected, smoked, snorted (past 6 months use and number of days).

- Other opioid use (pharmaceuticals and heroin): swallowed, injected, smoked, snorted (past 6 months use and number of days).

- Street (diverted) price and perceived availability of diverted tapentadol and other opioids.

- Attractiveness of tapentadol for use via injection.

- Overdose (each opioid).

\section{Data analysis}

Population-level data

Opioid utilisation (IMS Health) and intentional pharmaceutical opioid exposure poisonings (PIC) data will be presented graphically over time by opioid type, opioid formulation and, where available tablet strength (nationally and by jurisdiction). Opioid utilisation data will be presented as number of packs and number of oral morphine equivalent (OME) grams; the latter will be computed using published conversion guidelines. ${ }^{25}$ Percentage of total OME/packs will be calculated for tapentadol and other opioid formulations to graph the underlying rate of sales. Trends over time in tapentadol sales will be assessed using negative binomial or generalised estimating equations (to take into account non-independence), including variables to account for time/ seasonality.

PIC data will be presented as rates of intentional pharmaceutical opioid exposure poisonings using IMS sales data as the denominator; specifically, rates per 100000
OME grams and per 1000 packs distributed per month. These analyses will be restricted to those cases explicitly identified as a pharmaceutical opioid exposure poisoning attributable to a type of opioid (eg, tapentadol, oxycodone) as per the criteria identified above. Trends over time in tapentadol exposures will also be modelled using generalised estimating equations.

Various comparators will be used for these analyses, including specific pharmaceutical opioid formulations (eg, tramadol, oxycodone), and opioids grouped as per WHO guidelines ${ }^{26}$ into strong prescription opioids (buprenorphine, fentanyl, hydromorphone, methadone, morphine and oxycodone; all listed as 'Schedule 8 ' opioids in Australian classification of medicines and poisons), other prescription opioids (prescribed codeine, dextropropoxyphene and tramadol; 'Schedule 4' in Australia) and over-the-counter opioids (codeine products available at pharmacies without a prescription).

\section{Sentinel data}

Cryptomarket and IDRS data will be described using descriptive statistics, with the former graphed as number of vendors for SRF tapentadol and other pharmaceutical opioid formulations by month. Parametric and non-parametric tests of significance, as well as measures of effect size, will be used to describe differences in rates of extramedical use, diversion, attractiveness and overdose for tapentadol versus other opioid formulations.

\section{ETHICS AND DISSEMINATION}

The authors confirm ethics approval is not required for pharmaceutical sales data. Ethics approval has been obtained for use of national pharmaceutical opioid poisonings reported to Poison Information Centres (LNR/16/SCHN/44) and for use of online marketplace data and interview data from people who inject drugs (HC13081 and HC12086). IDRS participants provided informed consent prior to completing the interview.

We plan to publish our findings mid-2018 in a peer-reviewed journal article as per the data sources and outcomes listed in table 1 and research questions specified in the aims above, and these findings will also be presented at conferences. Publications will comply with Strengthening the Reporting of Observational Studies in Epidemiology guidelines. ${ }^{27}$ Restrictions will apply to the availability of these data as they are used with approval from data custodians for the purposes of this study, but they may be available following approval from the researchers, custodians of the data and any other involved third party.

\section{DISCUSSION}

This postmarketing study forms part of a growing body of literature detailing comprehensive and transparent monitoring of specific pharmaceutical opioid formulations with key indicators of use, extramedical use and harms. ${ }^{28}$ Concern is growing regarding rates of 
pharmaceutical opioid extramedical use and harms. Consequently, it is important to determine how shifts in the pharmaceutical opioid market, including availability of new formulations, plays out in terms of these outcomes. There has been no such undertaking for tapentadol globally, despite availability in the US for nearly a decade, and indications of a small but growing pharmaceutical opioid market share, ${ }^{17}$ making the current study critical.

It should be noted that this study is limited in terms of the number of available data sources relative to other postmarketing studies. ${ }^{28}$ However, this is a systems-level issue, as tapentadol is not differentiated from other pharmaceutical opioids in most healthcare and law enforcement data coding systems. There are a number of limitations specific to the chosen data sources. IDRS reflects a sentinel population at high risk of extramedical use, and thus will not reflect general population extramedical use, nor will it cover all aspects of various outcomes (eg, street price is only one aspect of pharmaceutical opioid attractiveness for extramedical use). Further, IMS Health sales data does not index the number of individuals prescribed opioids, and PIC data does not index all individuals with an adverse event, the latter often captured in other healthcare sources. However, IMS data is estimated to cover over $94 \%$ of all sales nationally, ${ }^{29}$ making it strong index of pharmaceutical opioid exposure in the community. Similarly, PIC data has complete coverage nationally and captures details related to type of exposure and dose critical to understanding risk of adverse exposure. We would also argue that online drug marketplace data and IDRS are necessary and key sentinel data sources, being established to identify emerging trends in drug use. IDRS participants typically report injecting heroin, methamphetamine and/or pharmaceutical opioids, ${ }^{24}$ making valid comparison across these substances and across jurisdictions with varying levels of heroin and pharmaceutical opioid availability.

In light of these strengths, we think that triangulation of these sources will provide a clear picture of tapentadol use, extramedical use, attractiveness and associated harms. We believe these findings will be critical to understanding possible risks in terms of extramedical use and harms if tapentadol prescribing increases. More broadly though, these findings will help to enhance understanding as to the impact changes in the pharmaceutical opioid market can have on extramedical use and harms from a policy, industry, clinician and research perspective.

Acknowledgements Thank you to Jared Brown of the NSW Poison Information Centre for his advice on the study protocol.

Contributors LD, AP, BL, MF, NB and RC contributed to the development of the study for the purposes of the funding proposal and development of the study design. LD, AP and BL led writing of the first draft. All authors contributed to the critical review of the manuscript. All authors read and approved the final manuscript.

Funding This work was supported by investigator-initiated untied educational funding from Seqirus Pty Ltd (the marketer of tapentadol SRF in Australia) granted to $A P, B L, M F, R C$, and $L D . B L, A P$ and $L D$ are supported by NHMRC research fellowships (\#1073858, \#1109366 and \#1041472). The National Drug and Alcohol Research Centre at UNSW Australia is supported by funding from the Australian Government under the Substance Misuse Prevention and Service Improvements Grant Fund.

Disclaimer The funder had no role in the design, conduct or interpretation of the study's findings.

Competing interests Some of the investigators have received investigatorinitiated untied educational grants from Reckitt Benckiser/Indivior for studies of buprenorphine-naloxone (BL, LD), buprenorphine depot (BL, LD, MF), naloxone (LD, MF), the development of an opioid-related behavior scale (BL, LD), the pharmacogenetic predictors of treatment success (RA) and a study of opioidsubstitution therapy uptake among patients with chronic non-cancer pain (BL, LD). Some of the investigators have also received investigator-initiated untied educational grants from Mundipharma for postmarketing surveillance of a tamperresistant opioid formulation (AP, BL, LD, MF).

Patient consent Detail has been removed from this case description/these case descriptions to ensure anonymity. The editors and reviewers have seen the detailed information available and are satisfied that the information backs up the case the authors are making.

Ethics approval Ethics approval for use of online marketplace data and interview data from people who inject drugs has been granted by the University of New South Wales Human Research Ethics Committee (HC12086), as well as from the relevant ethics committees in each jurisdiction. The Sydney Children's Hospitals Network Human Research Ethics Committee has approved the use of data collected by the PIC (retrospective review of incidence and toxicity of various exposures from calls to Australian Poisons Information Centres; LNR/16/SCHN/44).

Provenance and peer review Not commissioned; externally peer reviewed.

Open Access This is an Open Access article distributed in accordance with the Creative Commons Attribution Non Commercial (CC BY-NC 4.0) license, which permits others to distribute, remix, adapt, build upon this work non-commercially, and license their derivative works on different terms, provided the original work is properly cited and the use is non-commercial. See: http://creativecommons.org/ licenses/by-nc/4.0/

(c) Article author(s) (or their employer(s) unless otherwise stated in the text of the article) 2018. All rights reserved. No commercial use is permitted unless otherwise expressly granted.

\section{REFERENCES}

1. Karanges EA, Blanch B, Buckley NA, et al. Twenty-five years of prescription opioid use in Australia: a whole-of-population analysis using pharmaceutical claims. Br J Clin Pharmacol 2016;82:255-67.

2. Blanch B, Pearson SA, Haber PS. An overview of the patterns of prescription opioid use, costs and related harms in Australia. $\mathrm{Br} \mathrm{J}$ Clin Pharmacol 2014;78:1159-66.

3. Larance B, Degenhardt L, Lintzeris N, et al. Definitions related to the use of pharmaceutical opioids: extramedical use, diversion, nonadherence and aberrant medication-related behaviours. Drug Alcohol Rev 2011;30:236-45.

4. Compton WM, Volkow ND. Major increases in opioid analgesic abuse in the United States: concerns and strategies. Drug Alcohol Depend 2006;81:103-7.

5. Compton WM, Volkow ND. Abuse of prescription drugs and the risk of addiction. Drug Alcohol Depend 2006;83:S4-7.

6. Drewes AM, Jensen RD, Nielsen LM, et al. Differences between opioids: pharmacological, experimental, clinical and economical perspectives. Br J Clin Pharmacol 2013;75:60-78.

7. US Food and Drug Administration. FDA opioids action plan. 2016 http://www.fda.gov/NewsEvents/Newsroom/FactSheets/ucm484714. htm

8. Manchikanti L, Abdi S, Atluri S, et al. American Society of Interventional Pain Physicians (ASIPP) guidelines for responsible opioid prescribing in chronic non-cancer pain: Part 2--guidance. Pain Physician 2012;15:S67-116.

9. Kress HG. Tapentadol and its two mechanisms of action: is there a new pharmacological class of centrally-acting analgesics on the horizon? Eur J Pain 2010;14:781-3.

10. Riemsma R, Forbes C, Harker J, et al. Systematic review of tapentadol in chronic severe pain. Curr Med Res Opin 2011;27:1907-30. 
11. World Health Organisation. Tapentadol: critical review report. Geneva: World Health Organisation, 2014.

12. Vorsanger G, Xiang J, Okamoto A, et al. Evaluation of study discontinuations with tapentadol inmmediate release and oxycodone immediate release in patients with low back or osteoarthritis pain. $J$ Opioid Manag 2010;6:169-79.

13. Vadivelu N, Timchenko A, Huang Y, et al. Tapentadol extendedrelease for treatment of chronic pain: a review. J Pain Res 2011;4:211.

14. Butler SF, McNaughton EC, Black RA. Tapentadol abuse potential: a postmarketing evaluation using a sample of individuals evaluated for substance abuse treatment. Pain Med 2015;16:119-30.

15. Cepeda MS, Fife D, Kihm MA, et al. Comparison of the risks of shopping behavior and opioid abuse between tapentadol and oxycodone and association of shopping behavior and opioid abuse. Clin J Pain 2014;30:1051-6.

16. McNaughton EC, Black RA, Weber SE, et al. Assessing abuse potential of new analgesic medications following market release: an evaluation of Internet discussion of tapentadol abuse. Pain Med 2015;16:131-40.

17. Cepeda MS, Fife D, Ma Q, et al. Comparison of the risks of opioid abuse or dependence between tapentadol and oxycodone: results from a cohort study. J Pain 2013;14:1227-41.

18. Dart RC, Cicero TJ, Surratt HL, et al. Assessment of the abuse of tapentadol immediate release: the first 24 months. J Opioid Manag 2012;8:395-402.

19. Perglozzi JV, Schug SA, Raffa RB, et al. Tapentadol and dual pain inhibition: a new strategy for pain relief in Australia. International Journal of Chronic Diseases 2015:1011-8.

20. Pharmaceutical Benefits Scheme. Tapentadol, tablet, $50 \mathrm{mg}, 100 \mathrm{mg}$, $150 \mathrm{mg}, 200 \mathrm{mg}$ and $250 \mathrm{mg}$ (as hydrochloride) (sustained release),
Palexia SR®. 2013 http://www.pbs.gov.au/info/industry/listing/ elements/pbac-meetings/psd/2013-11/tapentadol

21. Cairns R, Brown JA, Buckley NA. The impact of codeine rescheduling on misuse: a retrospective review of calls to Australia's largest poisons centre. Addiction 2016;111:1848-53.

22. Burns $L$, Roxburgh $A$, Bruno $R$, et al. Monitoring drug markets in the Internet age and the evolution of drug monitoring systems in Australia. Drug Test Anal 2014;6:840-5.

23. Roxburgh A, Van Buskirk J, Burns L, et al. Drugs and the Internet. Sydney: National Drug and Alcohol Research Centre, 2017.

24. Stafford J, Breen C. Australian Drug Trends 2016: Findings from the Illicit Drug Reporting System (IDRS). Sydney: National Drug and Alcohol Research Centre, 2017.

25. Nielsen S, Degenhardt L, Hoban B, et al. Comparing opioids: A guide to estimating oral morphine equivalents (OME) in research. Sydney: National Drug and Alcohol Research Centre, 2014.

26. Zech DF, Grond S, Lynch J, et al. Validation of World Health Organization Guidelines for cancer pain relief: a 10-year prospective study. Pain 1995;63:65-76.

27. Vandenbroucke JP, von Elm E, Altman DG, et al. Strengthening the Reporting of Observational Studies in Epidemiology (STROBE): explanation and elaboration. PLoS Med 2007;4:e297.

28. Larance B, Dobbins T, Peacock A, et al. The effect of a potentially tamper-resistant oxycodone formulation on opioid use and harm: main findings of the National Opioid Medications Abuse Deterrence (NOMAD) study. Lancet Psychiatry 2018;5:155-66.

29. Health IMS. Australian Pharmaceutical Index: IMS Plus workshop manual. Sydney: IMS Health Australia, 2009. 